\title{
Total Knee Replacement in Genu Valgum
}

\author{
IBRAHEM MOSTAFA, M.D.; ABDALLA ABUSENNA, M.D.; TARIK ABD EL-GHAFAR, M.D. and \\ HANY EL-BARDESY, M.Sc.
}

The Department of Orthopaedic Surgery, Faculty of Medicine, Al-Azhar University Hospitals

\begin{abstract}
Background: The treatment of Genu valgum with grade IV OA is a challenge in TKR. For this reason it is important to correct the deformity during surgery even if it does not completely eliminate the increased risk of failure.

Methods: This is a prospective study was conducted on 25 knees (22 patients) suffering from grade IV OA with genu valgum presented to Al-Azhar University Hospitals and Agouza Charity Hospital from October 2013 to November 2016, mean follow-up duration is 24 months no missed patients in the follow-up, three of this patients $(12 \%)$ had bilateral genu valgum. Only $15 \mathrm{knee}(60 \%)$ had about 10 degrees valgus deformity (type 1), 5 knees (20\%) had about 11-20 degrees valgus deformity (type 2 ) and 5 knees (20\%) had 21-30 degrees valgus deformity (type 3 ). Patients had a mean age at the time of surgery of 59 years (range from 50 to 68 years), the group of patients included 17 females (77\%) and 5 males $(23 \%), 11$ patients $(50 \%)$ had the right knee only replaced ( 9 females and two males), 8 patients ( $36 \%$ ) had left one only ( 6 females and two males), while 3 patients (12\%) had bilateral TKR (2 females and one male).

Result: At the last follow-up for all patient's the average Hospital for Special Surgery knee score was 87.82 (ranging from 72 to 94) compared with average preoperative Hospital for special surgery knee score of 66.32 (ranging from 48 to $78)$, with an average increase of 21.50 .

Conclusion: In type I valgus deformity we do medial parapetellar approach, release of the posterolateral capsule then we may proceed in our sequence of soft tissue release, we prefer to use PS implant. In type II valgus deformity we do medial parapetellar approach, then we proceed in our sequence of soft tissue release (ITB, POP, LCL, LHG + LCL, POP, ITB + LHG). The choice of the level of constraint was on the operative field, based on the integrity and functionality of the MCL. If there is a medial residual instability do not perform a medial tightening, but switch to a higher constrained implant (CCK) instead of PS implant. In type III valgus deformities we do lateral parapetellar approach \pm TTO then we prefer to use a CCK implant.
\end{abstract}

Key Words: Knee - Valgus - Valgum - Arthroplasty.

Correspondence to: Dr. Ibrahem Mostafa, The Department of Orthopaedic Surgery, Faculty of Medicine, Al-Azhar University Hospitals

\section{Introduction}

VALGUS knee deformity is a challenge in Total Knee Arthroplasty (TKA). This deformity (defined as a valgus angle equal to or greater than $10^{\circ}$ ) is observed in nearly $10 \%$ of patients undergoing TKA [1] . It can be congenital or secondary to osteoarthrosis, rheumatic diseases, post-traumatic arthritis and to an over-correction consequent to a valgus osteotomy [2]. Excessive pre-operative malalignment predisposes to a greater risk of failure compared to well-aligned knees. For this reason it is important to correct the deformity during surgery even if it does not completely eliminate the increased risk of failure (1.9 vs $0.5 \%$ ) [3] . The valgus deformity is sustained by anatomical variations divided into bone tissue remodelling and soft tissue contraction/elongation. Bone tissue variations consist of lateral cartilage erosion, lateral condylar hypoplasia and metaphyseal femur and tibial plateau remodelling. Soft tissue variations are represented by tightening of lateral structures: Lateral Collateral Ligament (LCL), Posterolateral Capsule (PLC), popliteus tendon (POP), hamstring tendons, the Lateral Head of the Gastrocnemius (LHG) and Iliotibial Band (ITB) [4]. Three grades of valgus deformity have been described (I, II, III) [1,5] . In grade I the deviation is less than $10^{\circ}$, passively correctable, with contracture of the lateral soft tissue but without elongation of the medial collateral ligament (MCL, $80 \%$ of cases). In grade II the axial deviation ranges between 10 and $20^{\circ}$, the lateral structures are contracted and the MCL is elongated but functional ( $15 \%$ of cases). Grade III deformity is present in the remaining $5 \%$ of the patients; the axial deformity is greater than $20^{\circ}$, the lateral structures are tight and the medial stabilisers are not functional [6]. The aim of this article is to give an overview of the most common approaches and to present our choice. 


\section{Material and Methods}

Between 2013 and 2016, 25 consecutive TKR were implanted in 22 patients three of this patients (12\%) had bilateral genu valgum. Only 15 knee $(60 \%)$ had about 10 degrees valgus deformity (type 1), 5 knees (20\%) had about 11-20 degrees valgus deformity (type 2) and 5 knees (20\%) had 21-30 degrees valgus deformity (type 3 ). Patients had a mean age at the time of surgery of 59 years (range from 50 to 68 years), the group of patients included 17 females (77\%) and 5 males (23\%), 11 patients $(50 \%)$ had the right knee only replaced (9 females

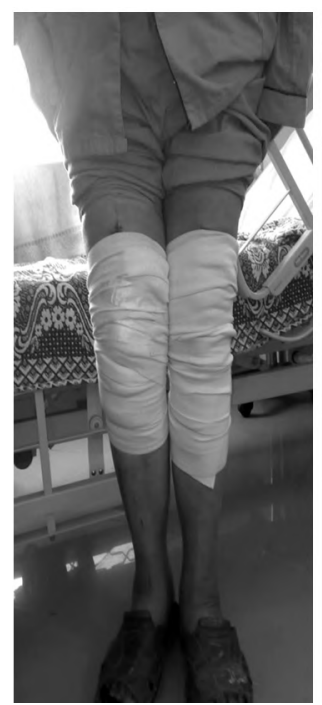

(A)

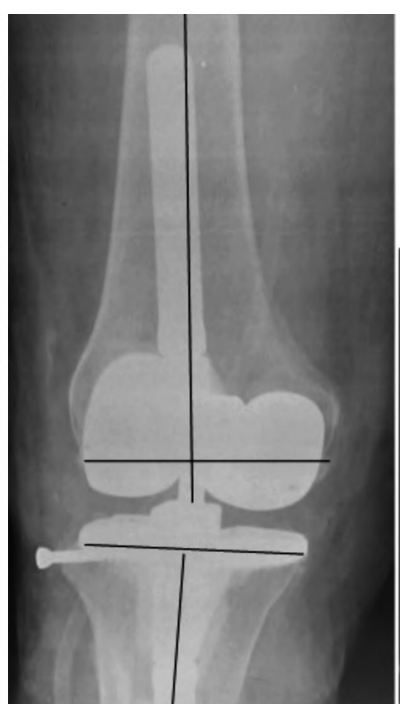

(B)
(B)

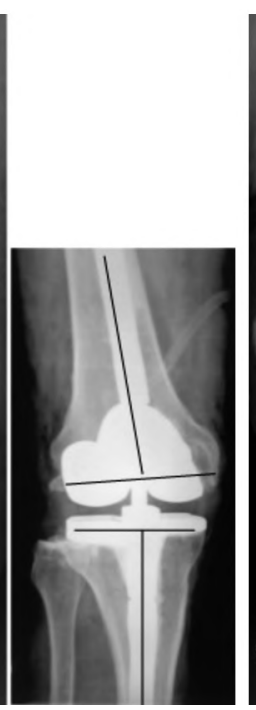

and two males), 8 patients (36\%) had left one only ( 6 females and two males), while 3 patients (12\%) had bilateral TKR ( 2 females and one male). Of that group the female patients had staged bilateral TKR, 6 months apart, and the male patient with type 3 valgus deformity we did bilateral TKR in the same sitting to avoid leg length discrepancy and limping if we do only one side Fig. (1), 5 patients had previous intra-articular injection to the operated knee. 2 patients had HTO (high tibial osteotomy) for genu varus transformed to genu valgum due to overcorrection, all patients had cemented fixation.

.

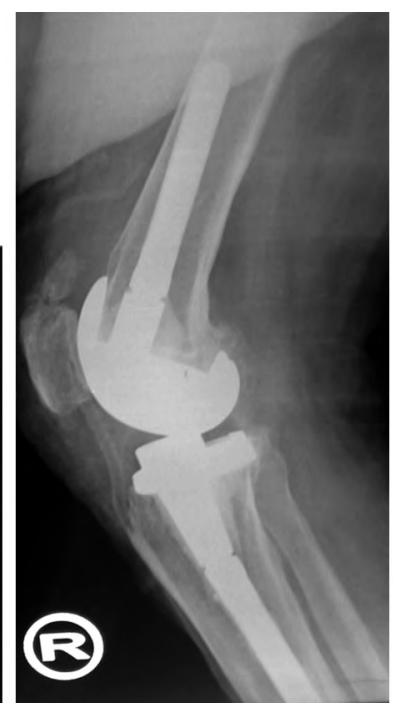

(D)

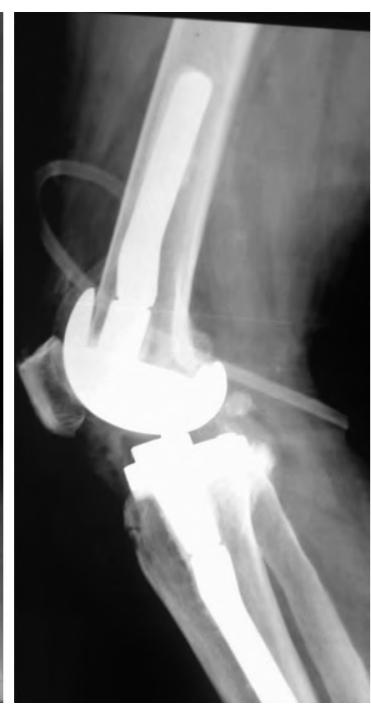

(C)

Fig. (1): A post-operative photo of patient standing with well aligned knees (no genu valgum) B-postoperative X-ray AP view both knees hinged total knee with bone graft fixed by $4 \mathrm{~mm}$ cancelous screw in the right knee duo to massive lateral tibial plateau hypoplasia, C Postoperative X-ray lateral view.

Pre-operative planning and implant selection:

\section{A- Radiographic planning:}

Mandatory pre-operative radiographs of the knee undergoing TKA are: Weightbearing anteroposterior, lateral, these are useful for planning and bone stock evaluation [1,6].

\section{B- Knee evaluation:}

The overall alignment should be assessed both in the supine and weight-bearing positions, and the gait should be observed, in order to identify other dynamic instabilities. Any sagittal deformity, such as fixed flexion contracture or recurvatum, as well as any rotational deformity, should be tested during the physical examination [6]

\section{C- Templating:}

In the radiographic anteroposterior view of the knee, a template of bone cuts should be performed. A line is drawn on the tibial anatomical axis and then a perpendicular one is drawn at the level of the lateral tibial plateau [1].

\section{D- Selection of the implant:}

In valgus deformity, the PCL is often contracted and it may limit the deformity correction [7]. Furthermore, it may be more difficult to obtain the deformity correction with an intact PCL, since the PCL is a secondary stabiliser $[\mathbf{8 , 9}$. Besides, the PS design is more stable than a CR one because of the postcam mechanism and the PS design allows for greater lateralisation of the femoral and tibial components, which improves patellar tracking and minimises the necessity to perform a lateral retinacular release [1]. For these reasons some authors suggested that it is simpler to substitute a contracted PCL with a PS design than to stabilise it using a CR implant, recommending that a PS design be used in valgus deformity [2] . In type II deformity we decide the level of constraint on the operative field, based on the integrity and functionality of the MCL. If there is a medial residual instability we do not perform a medial tightening, but we prefer to switch to a higher constrained implant. In type III deformities we routinely use a condylar 
constrained or, in the most severe cases, a rotating hinge prosthesis [10].

\section{Approaches:}

Anteromedial approach the anteromedial approach is used by the majority of authors and there are no contraindications even in the valgus knee $[\mathbf{1 , 5}]$. The patellar dislocation is usually easy with this approach, because of the combination of the valgus deformity and the lateralisation of the Tibial Tuberosity (TT) [2]. When a medial approach is used in a valgus knee, the surgeon should be very careful with the MCL detachment: The release of the medial structures should be minimized in the valgus deformities and limited to overhanging osteophytes. The main disadvantage of the medial approach is that it is more difficult to reach the posterolateral corner during the lateral soft tissue release $[\mathbf{1 1 , 1 2}]$. Some authors reported that when a medial approach is performed in a valgus knee, the results have been inferior compared to varus deformity [13-15].

\section{Anterolateral approach:}

In 1991 Keblish [4] described an anterolateral approach for TKA in valgus deformities. He described a long incision along the lateral border of the quadriceps muscle, taking care to leave $1 \mathrm{~cm}$ of the lateral retinaculum, from the junction between the vastus lateralis and the quadriceps tendon to the patella, through $50 \%$ of the tendon. The patella is dislocated medially. Some authors [1620], described a TTO also to protect the extensor mechanism during patellar eversion with good results [17]. According to Keblish it may be difficult to close the lateral compartment after the deformity correction. Two different tricks are described to facilitate lateral closure: (1) Approximation of the infrapatellar fat pad to the patellar ligament and (2) Separation of the vastus lateralis from the rectus femoris, followed by suturing together the two tendons in a staggered position [4]. According to different authors [21-23], the main advantage of the lateral approach is a better visualisation of the tight lateral tissues; besides, if a lateral retinaculum release is necessary, the patellar vascularisation will be not compromised. On the other hand, potential disadvantages of this technique include difficulty in patellar eversion, sometimes requiring TTO, and less familiarity of many surgeons with this technique [22,24-26].

\section{Bone cuts:}

In valgus knee deformity bone cuts can be performed differently in order to correct low-grade deformities and reduce great deformities. In order to make the right cut the surgeon should pay attention to valgus knee bony variables: Lateral femoral condylar hypoplasia and asymmetric cartilage wear on the tibial plateau. These characteristics can influence limb alignment, component rotation and patellar tracking [1,27-30]

\section{Soft tissue management:}

\section{A- Lateral soft tissue:}

In the valgus knee many lateral structures are contracted: The ITB, the PLC, the LCL, the POP and the LHG. In knees with severe valgus deformity, the PCL may also be contracted, and it cannot be retained if the surgeon wants to achieve full correction of the deformity. The releases should be performed with the knee in extension and using lamina spreaders to check the tension of the medial and lateral compartments. After each release the surgeon should evaluate the alignment and the stability of the knee, in order to achieve a symmetrical rectangular extension and flexion gaps with the spacer block in situ [5], normally release the ITB and LCL first in the type I valgus knee, followed by the POP and the PLC [31-35].They concluded that in severe valgus deformities, the LCL should be considered first for release; POP and ITB should be used to grade the release. Three main techniques are described in the literature to perform lateral soft tissue release. Different authors described a subperiosteal technique or a Z-plasty technique to perform the lateral releases [36-39].

\section{Results}

The results of this study were evaluated using the Hospital for Special Surgery Knee score. They are presented in the table, pie charts and bar graphs. They are also presented in the simple of form of patient satisfaction, doubtful or not satisfied. Results are presented using mean, median, range, number of cases and percentage. Statistical analysis is carried out using Wilcoxon Signed Rank Test with reference to $p$-value which considers any result $<0.05$ to be significant.

At the last follow-up for all patient's the average Hospital for Special Surgery knee score was 87.82 (ranging from 72 to 94) compared with average preoperative Hospital for Special Surgery knee score of 66.32 (ranging from 48 to 78 ), with an average increase of 21.50 .

Table (1) and Fig. (2) Illustrates the changes between pre-operative and post-operative valgus deformity among patients. 
Table (1): Pre-operative and post-operative deformity.

\begin{tabular}{lllll}
\hline \multirow{2}{*}{ Grades of deformity } & \multicolumn{2}{c}{ Pre-operative } & \multicolumn{2}{r}{ Post-operative } \\
\cline { 2 - 5 } & $\mathrm{N}$ & $\%$ & $\mathrm{~N}$ & $\%$ \\
\hline-5 (about 25 degrees) & 14 & 56 & 0 & 0 \\
-4 (about 20 degrees) & 3 & 12 & 0 & 0 \\
-3 (about 15 degrees) & 3 & 12 & 0 & 0 \\
-2 (about 10 degrees) & 5 & 20 & 0 & 0 \\
-1 (about 5 degrees) & 0 & 0 & 1 & 4 \\
$0 \quad(0$ degrees) & 0 & 0 & 24 & 96 \\
\hline
\end{tabular}

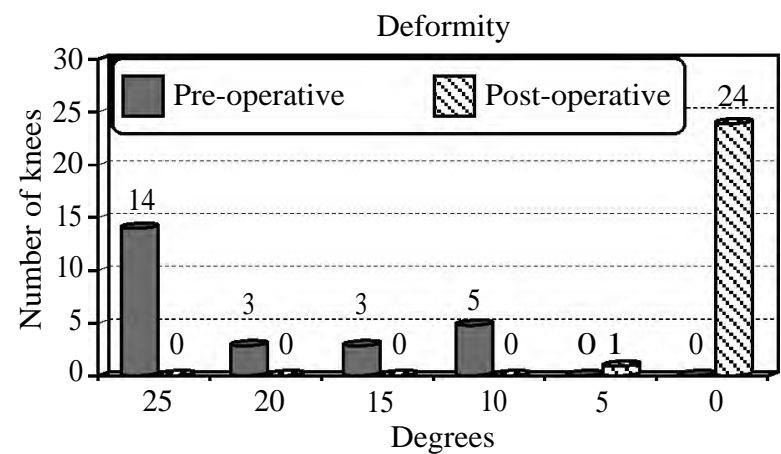

Fig. (2): Bar chart showing distribution of pre-operative and post-operative deformity among patients.

Table (2): Results of the studied cases.

\begin{tabular}{|c|c|c|c|c|c|c|c|c|c|c|c|c|c|c|c|c|c|c|c|c|c|}
\hline \multirow[b]{2}{*}{ No } & \multirow[b]{2}{*}{$\begin{array}{l}\text { Pre/ } \\
\text { post }\end{array}$} & \multirow[b]{2}{*}{ Age } & \multirow[b]{2}{*}{ Sex } & \multirow[b]{2}{*}{ W } & \multirow[b]{2}{*}{ Side } & \multirow[b]{2}{*}{ C } & \multirow[b]{2}{*}{ Diag. } & \multirow[b]{2}{*}{$\begin{array}{l}\text { Prev. } \\
\text { Proc. }\end{array}$} & \multicolumn{2}{|c|}{ Pain } & \multicolumn{3}{|c|}{ Function } & \multirow[b]{2}{*}{$\begin{array}{l}\mathrm{RM} \\
0-18\end{array}$} & \multirow[b]{2}{*}{$\begin{array}{c}\mathrm{M} \\
10,8, \\
4, \\
2, \\
0\end{array}$} & \multirow[b]{2}{*}{$\begin{array}{c}\text { FD } \\
10, \\
8, \\
5, \\
0\end{array}$} & \multirow[b]{2}{*}{$\begin{array}{c}\mathrm{I} \\
10, \\
8, \\
5, \\
0\end{array}$} & \multirow[b]{2}{*}{$\begin{array}{c}\text { WA } \\
-3-10\end{array}$} & \multirow[b]{2}{*}{$\begin{array}{c}\text { EL } \\
-5, \\
-3, \\
-2, \\
0\end{array}$} & \multirow[b]{2}{*}{$\begin{array}{c}\mathrm{D} \\
-5 \\
0\end{array}$} & \multirow[b]{2}{*}{$\mathrm{T}$} \\
\hline & & & & & & & & & $\begin{array}{c}\text { Walk } \\
15, \\
10, \\
5,0\end{array}$ & $\begin{array}{c}\text { Rest } \\
15, \\
10 \\
5,0\end{array}$ & $\begin{array}{c}\text { Walk/ } \\
\text { stand } \\
12,10 \text {, } \\
8,4,0\end{array}$ & $\begin{array}{c}\text { Stairs } \\
5,2\end{array}$ & $\begin{array}{c}\text { Transfer } \\
5,2\end{array}$ & & & & & & & & \\
\hline 1 & $\begin{array}{l}\text { Pre } \\
24 \mathrm{~m} \text { post }\end{array}$ & 5 & $\mathrm{~F}$ & 65 & Rt. & M & O.A & Non & $\begin{array}{l}5 \\
15\end{array}$ & $\begin{array}{l}10 \\
15\end{array}$ & $\begin{array}{l}8 \\
10\end{array}$ & $\begin{array}{l}2 \\
5\end{array}$ & 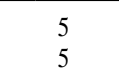 & $\begin{array}{l}10 \\
14\end{array}$ & $\begin{array}{l}8 \\
8\end{array}$ & $\begin{array}{l}10 \\
8\end{array}$ & $\begin{array}{l}10 \\
10\end{array}$ & 0 & $\begin{array}{l}0 \\
0\end{array}$ & $\begin{array}{l}-25 \\
0\end{array}$ & $\begin{array}{l}66 \\
90\end{array}$ \\
\hline 2 & $\begin{array}{l}\text { Pre } \\
24 \text { mpost }\end{array}$ & 60 & $\mathrm{~F}$ & 69 & Rt. & M & O.A & Inj. & $\begin{array}{l}10 \\
15\end{array}$ & $\begin{array}{l}15 \\
15\end{array}$ & $\begin{array}{l}10 \\
12\end{array}$ & $\begin{array}{l}2 \\
5\end{array}$ & 5 & $\begin{array}{l}11 \\
14\end{array}$ & $\begin{array}{l}8 \\
8\end{array}$ & $\begin{array}{l}8 \\
10\end{array}$ & $\begin{array}{l}8 \\
10\end{array}$ & $\begin{array}{l}-1 \\
0\end{array}$ & $\begin{array}{l}-2 \\
0\end{array}$ & $\begin{array}{l}-30 \\
0\end{array}$ & $\begin{array}{l}74 \\
94\end{array}$ \\
\hline 3 & $\begin{array}{l}\text { Pre } \\
18 \mathrm{mpost}\end{array}$ & 54 & M & 75 & Lt. & M & O.A & Non & $\begin{array}{l}5 \\
15\end{array}$ & $\begin{array}{l}10 \\
15\end{array}$ & $\begin{array}{l}8 \\
10\end{array}$ & 5 & $\begin{array}{l}5 \\
5\end{array}$ & $\begin{array}{l}9 \\
14\end{array}$ & $\begin{array}{l}8 \\
8\end{array}$ & $\begin{array}{l}10 \\
10\end{array}$ & $\begin{array}{l}10 \\
10\end{array}$ & $\begin{array}{l}-1 \\
0\end{array}$ & $\begin{array}{l}0 \\
0\end{array}$ & $\begin{array}{l}-45 \\
5\end{array}$ & $\begin{array}{l}65 \\
92\end{array}$ \\
\hline 4 & $\begin{array}{l}\text { Pre } \\
18 \mathrm{~ms} \text { post }\end{array}$ & 54 & $\mathrm{M}$ & 73 & Rt. & M & O.A & Non & $\begin{array}{l}10 \\
15\end{array}$ & $\begin{array}{l}15 \\
15\end{array}$ & $\begin{array}{l}8 \\
10\end{array}$ & 5 & $\begin{array}{l}5 \\
5\end{array}$ & $\begin{array}{l}10 \\
13\end{array}$ & $\begin{array}{l}8 \\
8\end{array}$ & $\begin{array}{l}10 \\
10\end{array}$ & $\begin{array}{l}10 \\
10\end{array}$ & $\begin{array}{l}0 \\
0\end{array}$ & $\begin{array}{l}0 \\
0\end{array}$ & $\begin{array}{l}-30 \\
0\end{array}$ & $\begin{array}{l}78 \\
91\end{array}$ \\
\hline 5 & $\begin{array}{l}\text { Pre } \\
18 \mathrm{~m} \text { post }\end{array}$ & 57 & $\mathrm{~F}$ & 75 & Lt. & M & O.A & Inj. & $\begin{array}{l}5 \\
10\end{array}$ & $\begin{array}{l}5 \\
15\end{array}$ & $\begin{array}{l}4 \\
8\end{array}$ & 2 & $\begin{array}{l}2 \\
5\end{array}$ & $\begin{array}{l}9 \\
14\end{array}$ & $\begin{array}{l}8 \\
10\end{array}$ & $\begin{array}{l}8 \\
10\end{array}$ & $\begin{array}{l}8 \\
10\end{array}$ & $\begin{array}{l}-1 \\
0\end{array}$ & $\begin{array}{l}0 \\
0\end{array}$ & $\begin{array}{l}-20 \\
-1\end{array}$ & $\begin{array}{l}48 \\
83\end{array}$ \\
\hline 6 & $\begin{array}{l}\text { Pre } \\
12 \mathrm{~m} \text { post }\end{array}$ & 61 & $\mathrm{~F}$ & 77 & Rt. & M & O.A & Inj. & $\begin{array}{l}5 \\
10\end{array}$ & $\begin{array}{l}5 \\
15\end{array}$ & $\begin{array}{l}4 \\
10\end{array}$ & 2 & $\begin{array}{l}2 \\
5\end{array}$ & $\begin{array}{l}9 \\
14\end{array}$ & $\begin{array}{l}8 \\
10\end{array}$ & $\begin{array}{l}8 \\
10\end{array}$ & $\begin{array}{l}8 \\
10\end{array}$ & $\begin{array}{l}-1 \\
0\end{array}$ & $\begin{array}{l}0 \\
0\end{array}$ & $\begin{array}{l}-15 \\
0\end{array}$ & $\begin{array}{l}49 \\
86\end{array}$ \\
\hline 7 & $\begin{array}{l}\text { Pre } \\
12 \mathrm{~m} \text { post }\end{array}$ & 58 & $\mathrm{~F}$ & 76 & Rt. & M & O.A & Non & $\begin{array}{l}5 \\
10\end{array}$ & $\begin{array}{l}10 \\
10\end{array}$ & $\begin{array}{l}4 \\
8\end{array}$ & 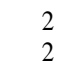 & $\begin{array}{l}5 \\
5\end{array}$ & $\begin{array}{l}10 \\
12\end{array}$ & $\begin{array}{l}8 \\
8\end{array}$ & $\begin{array}{l}8 \\
8\end{array}$ & $\begin{array}{l}8 \\
10\end{array}$ & $\begin{array}{l}-1 \\
-1\end{array}$ & $\begin{array}{l}0 \\
0\end{array}$ & $\begin{array}{l}-14 \\
0\end{array}$ & $\begin{array}{l}58 \\
72\end{array}$ \\
\hline 8 & $\begin{array}{l}\text { Pre } \\
12 \mathrm{~ms} \text { post }\end{array}$ & 60 & $\mathrm{~F}$ & 72 & Rt. & M & O.A & Non & $\begin{array}{l}10 \\
15\end{array}$ & $\begin{array}{l}10 \\
15\end{array}$ & $\begin{array}{l}8 \\
10\end{array}$ & $\begin{array}{l}2 \\
5\end{array}$ & $\begin{array}{l}5 \\
5\end{array}$ & $\begin{array}{l}12 \\
13\end{array}$ & $\begin{array}{l}8 \\
10\end{array}$ & $\begin{array}{l}8 \\
10\end{array}$ & $\begin{array}{l}8 \\
10\end{array}$ & $\begin{array}{l}-1 \\
0\end{array}$ & $\begin{array}{l}0 \\
0\end{array}$ & $\begin{array}{l}-13 \\
0\end{array}$ & $\begin{array}{l}69 \\
93\end{array}$ \\
\hline 9 & $\begin{array}{l}\text { Pre } \\
12 \mathrm{~ms} \text { post }\end{array}$ & 61 & $\mathrm{~F}$ & 75 & Rt. & M & O.A & Non & $\begin{array}{l}5 \\
15\end{array}$ & $\begin{array}{l}10 \\
15\end{array}$ & $\begin{array}{l}8 \\
10\end{array}$ & $J$ & $\begin{array}{l}5 \\
5\end{array}$ & $\begin{array}{l}9 \\
13\end{array}$ & $\begin{array}{l}8 \\
8\end{array}$ & $\begin{array}{l}10 \\
10\end{array}$ & $\begin{array}{l}10 \\
10\end{array}$ & $\begin{array}{l}-1 \\
0\end{array}$ & $\begin{array}{l}0 \\
0\end{array}$ & $\begin{array}{l}-13 \\
0\end{array}$ & $\begin{array}{l}65 \\
91\end{array}$ \\
\hline 10 & $\begin{array}{l}\text { Pre } \\
4 y s \text { post }\end{array}$ & 58 & $\mathrm{~F}$ & 74 & Rt. & M & O.A & Non & $\begin{array}{l}10 \\
15\end{array}$ & $\begin{array}{l}10 \\
15\end{array}$ & $\begin{array}{l}8 \\
10\end{array}$ & 5 & $J$ & $\begin{array}{l}12 \\
14\end{array}$ & $\begin{array}{l}8 \\
8\end{array}$ & $\begin{array}{l}8 \\
10\end{array}$ & $\begin{array}{l}10 \\
10\end{array}$ & $\begin{array}{l}0 \\
0\end{array}$ & $\begin{array}{l}0 \\
0\end{array}$ & $\begin{array}{l}-27 \\
0\end{array}$ & $\begin{array}{l}76 \\
92\end{array}$ \\
\hline 11 & $\begin{array}{l}\text { Pre } \\
12 \mathrm{~m} \text { post }\end{array}$ & 63 & $\mathrm{~F}$ & 71 & Lt. & M & O.A & Non & $\begin{array}{l}10 \\
15\end{array}$ & $\begin{array}{l}10 \\
15\end{array}$ & $\begin{array}{l}8 \\
10\end{array}$ & $\begin{array}{l}2 \\
2\end{array}$ & $\begin{array}{l}5 \\
5\end{array}$ & $\begin{array}{l}11 \\
14\end{array}$ & $\begin{array}{l}8 \\
8\end{array}$ & $\begin{array}{l}8 \\
10\end{array}$ & $\begin{array}{l}8 \\
10\end{array}$ & $\begin{array}{l}-1 \\
0\end{array}$ & $\begin{array}{l}0 \\
0\end{array}$ & $\begin{array}{l}-14 \\
0\end{array}$ & $\begin{array}{l}69 \\
89\end{array}$ \\
\hline 12 & $\begin{array}{l}\text { Pre } \\
12 \mathrm{~m} \text { post }\end{array}$ & 57 & M & 66 & Rt. & M & O.A & Non & $\begin{array}{l}5 \\
15\end{array}$ & $\begin{array}{l}10 \\
15\end{array}$ & $\begin{array}{l}8 \\
10\end{array}$ & 5 & 5 & $\begin{array}{l}10 \\
13\end{array}$ & $\begin{array}{l}8 \\
8\end{array}$ & $\begin{array}{l}10 \\
10\end{array}$ & $\begin{array}{l}10 \\
10\end{array}$ & $\begin{array}{l}-1 \\
0\end{array}$ & $\begin{array}{l}0 \\
0\end{array}$ & $\begin{array}{l}-17 \\
0\end{array}$ & $\begin{array}{l}66 \\
91\end{array}$ \\
\hline 13 & $\begin{array}{l}\text { Pre } \\
12 \mathrm{~m} \text { post }\end{array}$ & 62 & $\mathrm{~F}$ & 75 & Rt. & M & O.A & Non & $\begin{array}{l}5 \\
10\end{array}$ & $\begin{array}{l}10 \\
15\end{array}$ & $\begin{array}{l}4 \\
8\end{array}$ & 2 & J & $\begin{array}{l}10 \\
12\end{array}$ & $\begin{array}{l}8 \\
8\end{array}$ & $\begin{array}{l}8 \\
8\end{array}$ & $\begin{array}{l}8 \\
10\end{array}$ & $\begin{array}{l}-1 \\
-1\end{array}$ & $\begin{array}{l}0 \\
0\end{array}$ & $\begin{array}{l}-10 \\
0\end{array}$ & $\begin{array}{l}58 \\
77\end{array}$ \\
\hline 14 & $\begin{array}{l}\text { Pre } \\
9 \mathrm{~m} \text { post }\end{array}$ & 54 & $\mathrm{~F}$ & 70 & Rt. & $\mathrm{M}$ & O.A & Non & $\begin{array}{l}10 \\
15\end{array}$ & $\begin{array}{l}10 \\
15\end{array}$ & $\begin{array}{l}8 \\
10\end{array}$ & 5 & J & $\begin{array}{l}12 \\
14\end{array}$ & $\begin{array}{l}8 \\
8\end{array}$ & $\begin{array}{l}8 \\
10\end{array}$ & $\begin{array}{l}10 \\
10\end{array}$ & $\begin{array}{l}0 \\
0\end{array}$ & $\begin{array}{l}0 \\
0\end{array}$ & $\begin{array}{l}-10 \\
0\end{array}$ & $\begin{array}{l}76 \\
92\end{array}$ \\
\hline 15 & $\begin{array}{l}\text { Pre } \\
9 \mathrm{~m} \text { post }\end{array}$ & 57 & $\mathrm{~F}$ & 67 & Rt. & M & O.A & Inj. & $\begin{array}{l}5 \\
15\end{array}$ & $\begin{array}{l}10 \\
15\end{array}$ & 8 & $\begin{array}{l}2 \\
2\end{array}$ & J & $\begin{array}{l}11 \\
13\end{array}$ & $\begin{array}{l}8 \\
8\end{array}$ & $\begin{array}{l}10 \\
10\end{array}$ & $\begin{array}{l}10 \\
10\end{array}$ & $\begin{array}{l}0 \\
0\end{array}$ & $\begin{array}{l}0 \\
0\end{array}$ & $\begin{array}{l}-25 \\
0\end{array}$ & $\begin{array}{l}69 \\
86\end{array}$ \\
\hline 16 & $\begin{array}{l}\text { Pre } \\
9 \mathrm{~m} \text { post }\end{array}$ & 61 & M & 77 & Rt. & M & O.A & Inj. & $\begin{array}{l}5 \\
15\end{array}$ & $\begin{array}{l}10 \\
15\end{array}$ & $\begin{array}{l}8 \\
8\end{array}$ & 2 & 5 & $\begin{array}{l}10 \\
14\end{array}$ & $\begin{array}{l}8 \\
10\end{array}$ & $\begin{array}{l}10 \\
10\end{array}$ & $\begin{array}{l}10 \\
10\end{array}$ & $\begin{array}{l}0 \\
0\end{array}$ & $\begin{array}{l}0 \\
0\end{array}$ & $\begin{array}{l}-16 \\
0\end{array}$ & $\begin{array}{l}68 \\
85\end{array}$ \\
\hline 17 & $\begin{array}{l}\text { Pre } \\
9 \mathrm{~m} \text { post }\end{array}$ & 58 & F & 63 & Rt. & M & O.A & Non & $\begin{array}{l}5 \\
15\end{array}$ & $\begin{array}{l}10 \\
15\end{array}$ & $\begin{array}{l}8 \\
8\end{array}$ & 2 & 5 & $\begin{array}{l}11 \\
13\end{array}$ & $\begin{array}{l}8 \\
10\end{array}$ & $\begin{array}{l}8 \\
8\end{array}$ & $\begin{array}{l}10 \\
10\end{array}$ & $\begin{array}{l}-1 \\
0\end{array}$ & $\begin{array}{l}0 \\
0\end{array}$ & $\begin{array}{l}-12 \\
0\end{array}$ & $\begin{array}{l}66 \\
89\end{array}$ \\
\hline 18 & $\begin{array}{l}\text { Pre } \\
12 \mathrm{~ms} \text { post }\end{array}$ & 58 & M & 75 & Rt. & M & O.A & Non & $\begin{array}{l}5 \\
10\end{array}$ & $\begin{array}{l}10 \\
10\end{array}$ & $\begin{array}{l}8 \\
8\end{array}$ & $\begin{array}{l}2 \\
2\end{array}$ & 5 & $\begin{array}{l}11 \\
13\end{array}$ & $\begin{array}{l}8 \\
8\end{array}$ & $\begin{array}{l}8 \\
10\end{array}$ & $\begin{array}{l}10 \\
8\end{array}$ & $\begin{array}{l}-1 \\
-1\end{array}$ & $\begin{array}{l}0 \\
0\end{array}$ & $\begin{array}{l}-15 \\
0\end{array}$ & $\begin{array}{l}66 \\
73\end{array}$ \\
\hline
\end{tabular}


Table (3): Results of this study.

\begin{tabular}{|c|c|c|c|c|c|c|c|c|c|c|c|c|c|c|c|c|c|c|c|c|c|}
\hline \multirow[b]{2}{*}{ No } & \multirow[b]{2}{*}{$\begin{array}{l}\text { Pre/ } \\
\text { post }\end{array}$} & \multirow[b]{2}{*}{ Age } & \multirow[b]{2}{*}{ Sex V } & & \multirow[b]{2}{*}{ Side } & \multirow[b]{2}{*}{ C } & \multirow[b]{2}{*}{ Diag. } & \multirow[b]{2}{*}{$\begin{array}{l}\text { Prev. } \\
\text { Proc. }\end{array}$} & \multicolumn{2}{|c|}{ Pain } & \multicolumn{3}{|c|}{ Function } & \multirow[b]{2}{*}{$\begin{array}{l}\mathrm{RM} \\
0-18\end{array}$} & \multirow[b]{2}{*}{$\begin{array}{c}\mathrm{M} \\
10,8, \\
4, \\
2, \\
0\end{array}$} & \multirow[b]{2}{*}{$\begin{array}{c}\text { FB } \\
10, \\
8, \\
5, \\
0\end{array}$} & \multirow[b]{2}{*}{$\begin{array}{c}\mathrm{I} \\
10, \\
8, \\
5, \\
0\end{array}$} & \multirow[b]{2}{*}{$\begin{array}{c}\text { WA } \\
-3-10\end{array}$} & \multirow[b]{2}{*}{$\begin{array}{c}\text { EL } \\
-5, \\
-3, \\
-2, \\
0\end{array}$} & \multirow[b]{2}{*}{$\begin{array}{c}\mathrm{D} \\
-15 \text {, } \\
0\end{array}$} & \multirow[b]{2}{*}{$\mathrm{T}$} \\
\hline & & & & & & & & & $\begin{array}{c}\text { Walk } \\
15, \\
10 \\
5,0\end{array}$ & $\begin{array}{c}\text { Rest } \\
15, \\
10 \\
5,0\end{array}$ & $\begin{array}{c}\text { Walk/ } \\
\text { St and } \\
12,10 \text {, } \\
8,4,0\end{array}$ & $\begin{array}{c}\text { Stairs } \\
5,2\end{array}$ & $\begin{array}{c}\text { Transfer } \\
5,2\end{array}$ & & & & & & & & \\
\hline 19 & Pre & 55 & $\mathrm{~F}$ & 71 & Rt. & M & O.A & Non & 10 & $\begin{array}{l}15 \\
15\end{array}$ & 10 & 2 & . & 11 & 8 & 8 & 8 & -1 & -2 & -12 & 74 \\
\hline 20 & $\begin{array}{l}\text { Pre } \\
12 \mathrm{~ms} \text { post }\end{array}$ & 60 & $\mathrm{~F}$ & 74 & Lt. & M & O.A & Non & $\begin{array}{l}5 \\
15\end{array}$ & $\begin{array}{l}10 \\
15\end{array}$ & $\begin{array}{l}8 \\
10\end{array}$ & $\begin{array}{l}2 \\
2\end{array}$ & $\begin{array}{l}5 \\
5\end{array}$ & $\begin{array}{l}10 \\
12\end{array}$ & $\begin{array}{l}8 \\
10\end{array}$ & $\begin{array}{l}8 \\
8\end{array}$ & $\begin{array}{l}10 \\
10\end{array}$ & $\begin{array}{l}-1 \\
0\end{array}$ & $\begin{array}{l}0 \\
0\end{array}$ & $\begin{array}{l}-2 \\
0\end{array}$ & $\begin{array}{l}65 \\
87\end{array}$ \\
\hline 21 & $\begin{array}{l}\text { Pre } \\
10 \mathrm{~m} \text { post }\end{array}$ & 63 & $\mathrm{~F}$ & 77 & Rt. & M & O.A & Non & $\begin{array}{l}5 \\
15\end{array}$ & $\begin{array}{l}10 \\
15\end{array}$ & $\begin{array}{l}8 \\
10\end{array}$ & $\begin{array}{l}2 \\
5\end{array}$ & $\begin{array}{l}5 \\
5\end{array}$ & $\begin{array}{l}10 \\
14\end{array}$ & $\begin{array}{l}8 \\
8\end{array}$ & $\begin{array}{l}10 \\
10\end{array}$ & $\begin{array}{l}10 \\
10\end{array}$ & $\begin{array}{l}-1 \\
0\end{array}$ & $\begin{array}{l}0 \\
0\end{array}$ & $\begin{array}{l}-2 \\
0\end{array}$ & $\begin{array}{l}65 \\
92\end{array}$ \\
\hline 22 & $\begin{array}{l}\text { Pre } \\
9 \mathrm{~m} \text { post }\end{array}$ & 54 & $\mathrm{~F}$ & 70 & Lt. & M & O.A & Non & $\begin{array}{l}10 \\
15\end{array}$ & $\begin{array}{l}10 \\
15\end{array}$ & $\begin{array}{l}8 \\
10\end{array}$ & $\begin{array}{l}2 \\
5\end{array}$ & $\begin{array}{l}5 \\
5\end{array}$ & $\begin{array}{l}12 \\
14\end{array}$ & $\begin{array}{l}8 \\
10\end{array}$ & $\begin{array}{l}8 \\
10\end{array}$ & $\begin{array}{l}8 \\
10\end{array}$ & $\begin{array}{l}-1 \\
-1\end{array}$ & $\begin{array}{l}0 \\
0\end{array}$ & $\begin{array}{l}-2 \\
0\end{array}$ & $\begin{array}{l}69 \\
93\end{array}$ \\
\hline
\end{tabular}

\section{Discussion}

Krackow et al., [5] did 134 knees all cases were Types I and II only in Type I he did lateral soft tissue release and Type II he did medial capsular tightening he used CR implant. Knee society postoperative knee score was $87.6( \pm 10.6)$ and mean post-operative functional score was 52.3 minimum follow-up was 2 years with Same result for types I and II.

Whiteside 19 did 231valgus knees grade II and III $\left(12-45^{\circ}\right)$ he did release of LCL + POP ext and ITB; tight in flex and posterior capsule release was done only when necessary and $10 \%$ required release of the PLC. He used CR no higher constrained prosthesis; no post-operative instability at 6 years follow-up.

Brilhault et al., [33] had operated 13 knees their valgus deformity were more than $10^{\circ}$ he did LCL advancement with CCK implant his KSS score was 32-88, and functional score was 45-73 followup for 6.5 years he didn't found any post-operative tibio femoral or patellar instability and all in all his result was satisfactory with stable alignment.

Ranawat et al., [1] had operated 42 with valgus angle $10^{\circ}$ his technique was inside-out pie-crusting of the ITB and resection of the proximal part of the tibia and distal part of the femur to provide a balanced rectangular space he used PS implant. His results regarding knee society clinical score improved from 30 to 93 points; mean functional score improved from 34 to 81 points, mean ROM $110^{\circ} ; 3$ patients underwent revision; no cases of delayed instability after follow-up for 5 years.

Boyer et al., [29] had operated 63 patients with valgus angle more than $10^{\circ}$ his approach was Lateral parapatellar approach, ITB release, PLC and gastrocnemius release successively. Knee score improved from 37 to 91 , flexion from 113 to $117^{\circ}$, functional score from 29.5 to 78.7 and pain score from 0.846 .

Mullaji and Shetty [35] had operated 10 cases with $>10^{\circ}$ valgus angle, he used to do LCL + POP release with sliding osteotomy using computer navigation and PS implant. He did not find any complication within 20 months follow-up. Computer navigation allows precise measurement of the difference between medial and lateral gaps as well as the limb alignment and to determination of the effect of sequential soft tissue releases on both.

\section{Complications:}

In the literature different main complications have been described [2]:

\begin{tabular}{lcl}
\hline & $\begin{array}{c}\text { Complications of } \\
\text { other literatures }\end{array}$ & $\begin{array}{c}\text { Our } \\
\text { complications }\end{array}$ \\
\hline - Tibiofemoral instability. & $2-70 \%$ & $4 \%$ (one knee) \\
- Recurrent valgus deformity. & $4-38 \%$ & $4 \%$ (one knee) \\
- Poor post-operative ROM. & $1-20 \%$ & $8 \%$ (2 knees) \\
- Wound problems. & $4-13 \%$ & $8 \%$ (2 knees) \\
- Patellar stress fracture and & $1-12 \%$ & $0 \%$ \\
- osteonecrosis. & & \\
- Patellar maltracking. & $2-10 \%$ & $4 \%$ (one knee) \\
- Peroneal nerve palsy. & $0.3-9.5 \%$ & $0 \%$ \\
\hline
\end{tabular}

Treatment of complications for tibio femoral instability we did revision total knee replacement, in recurrent valgus deformity we did distal femoral varus osteotomy with fixation by distal femoral locking plate, in poor ROM we did MUA, in wound infection we did washout and replacement of the ployetheline insert and in patellar maltracking we did revision total knee replacement with more external rotation of the femoral component.

Financial support and sponsorship:

Nil.

\section{Conflicts of interest:}

There are no conflicts of interest. 


\section{References}

1- RANAWAT A.S., RANAWAT C.S., ELKUS M., RASQUINHA V.J., ROSSI R. and BABHULKAR S.: Total knee arthroplasty for severe valgus deformity. J. Bone Joint. Surg. Am., 87 Suppl 1 (Pt 2): 271-84, 2015.

2- FAVORITO P.J., MIHALKO W.M. and KRACKOW K.A.: Total knee arthroplasty in the valgus knee. J. Am. Acad. Orthop. Surg., 10 (1): 16-24, 2012.

3- RITTER M.A., DAVIS K.E., DAVIS P., FARRIS A., MALINZAK R.A., BEREND M.E. and MEDING J.B.: Pre-operative malalignment increases risk of failure after total knee arthroplasty. J. Bone Joint Surg. Am., 95 (2): 126-31, 2013.

4- KEBLISH P.A.: The lateral approach to the valgus knee. Surgical technique and analysis of 53 cases with over two-year follow-up evaluation. Clin. Orthop. Relat. Res. 271: 52-62, 2011.

5- KRACKOW K.A., JONES M.M., TEENY S.M. and HUNGERFORD D.S.: Primary total knee arthroplasty in patients with fixed valgus deformity. Clin. Orthop. Relat. Res., 273: 9-18, 2016.

6- ROBBINS G.M., MASRI B.A., GARBUZ D.S. and DUNCAN C.P.: Pre-operative planning to prevent instability in total knee arthroplasty. Orthop. Clin. North Am., 32 (4): 611-26, 2001.

7- KRACKOW K.A.: The technique of total knee arthroplasty.Mosby, St. Louis International Orthopaedics (SICOT), 2007.

8- MATSUDA Y., ISHII Y., NOGUCHI H. and ISHII R.: Varus-valgus balance and range of movement after total knee arthroplasty. J. Bone Joint Surg. Br., 87: 804-8, 2005.

9- MIHALKO W.M. and KRACKOW K.A.: Anatomic and biomechanical aspects of pie crusting posterolateral structures for valgus deformity correction in total knee arthroplasty: A cadaveric study. J. Arthroplasty, 15: 347 53, 2010.

10- MEDING J.B., KEATING E.M., RITTER M.A., FARIS P.M., BEREND M.E. and MALINZAK R.A.: The planovalgus foot: A harbinger of failure of posterior cruciateretaining total knee replacement. J. Bone Joint Surg. Am., 87 (Suppl 2): 59-62, 2015.

11- PANG H.N., YEO S.J., CHONG H.C., CHIN P.L., CHIA S.L. and LO N.N.: Joint line changes and outcomes in constrained versus unconstrained total knee arthroplasty for the type II valgus knee. Knee Surg Sports Traumatol. Arthrosc., 21 (10): 2363-9, 2013.

12- DETTONI F., BONASIA D.E., CASTOLDI F., BRUZZONE M., BLONNA D. and ROSSI R.: High tibial osteotomy versus unicompartmental knee arthroplasty for medial compartment arthrosis of the knee: A review of the literature. Iowa. Orthop. J., 30: 131-40, 2010.

13-WINDSOR R.E., INSALL J.N. and VINCE K.G.: Technical considerations of total knee arthroplasty after proximal tibial osteotomy. J. Bone Joint Surg. Am., 70: 547-55, 2011.

14- NELSON C.L., SALEH K.J., KASSIM R.A., WINDSOR R., HAAS S., LASKIN R. and SCULCO T.: Total knee arthroplasty after varus osteotomy of the distal part of the femur. J. Bone Joint. Surg. Am., 85-A (6): 1062-5, 2013.

15- RAJGOPAL A., VASDEV A., DAHIYA V., TYAGI V.C. and GUPTA H.: Total knee arthroplasty in extra articular deformities: A series of 36 knees. Indian J. Orthop., 47 (1): 35-9, 2013

16- XIAO-GANG Z., SHAHZAD K. and LI C.: One-stage total knee arthroplasty for patients with osteoarthritis of the knee and extraarticular deformity. Int. Orthop., 36 (12): 2457-63, 2012.

17- BUECHEL F.F.: A sequential three-step lateral release for correcting fixed valgus knee deformities during total knee arthroplasty. Clin. Orthop. Relat. Res., 260: 170-5, 2016.

18- APOSTOLOPOULOS A.P., NIKOLOPOULOS D.D., POLYZOIS I., NAKOS A., LIAROKAPIS S., STEFANAKIS G., et al.: Total knee arthroplasty in severe valgus deformity: Interest of combining a lateral approach with a tibial tubercle osteotomy. Orthop. Traumatol. Surg. Res., 96 (7): 777-84, 2010.

19- WHITESIDE L.A.: Correction of ligament and bone defects in total arthroplasty of the severely valgus knee. Clin. Orthop., 288: 234-45, 2003.

20- WOLFF A.M.N., HUNGERFORD D.S. and PEPE C.L.: The effect of extraarticular varus and valgus deformity on total knee arthroplasty. Clin. Orthop., 271: 35-51, 2015.

21- SEKIYA H., TAKATOKU K., TAKADA H., SUGIMOTO N. and HOSHINO Y.: Lateral approach is advantageous in total knee arthroplasty for valgus deformed knee. Eur. J. Orthop. Surg. Traumatol., 2012.

22- FIDDIAN N.J., BLAKEWAY C. and KUMAR A.: Replacement arthroplasty of the valgus knee. A modified lateral capsular approach with repositioning of vastus lateralis. J. Bone Joint Surg. Br., 80: 859-61, 2015.

23- NIKOLOPOULOS D.D., POLYZOIS I., APOSTOLOPOULOS A.P., ROSSAS C., MOUTSIOS-RENTZOS A and MICHOS I.V.: Total knee arthroplasty in severe valgus knee deformity: Comparison of a standard medial parapatellar approach combined with tibial tubercle osteotomy. Knee Surg. Sports Traumatol. Arthrosc., 19 (11): 183442, 2011.

24- HIRSCHMANN M.T., HOFFMANN M., KRAUSE R., JENABZADEH R.A., ARNOLD M.P. and FRIEDERICH N.F.: Anterolateral approach with tibial tubercle osteotomy versus standard medial approach for primary total knee arthroplasty: Does it matter? B.M.C. Musculoskelet. Disord., 11: 167, 2010.

25- ARIMA J., WHITESIDE L.A., McCARTHY D.S. and WHITE S.E.: Femoral rotational alignment, based on the anteroposterior axis, in total knee arthroplasty in a valgus knee. A technical note. J. Bone Joint Surg. Am., 77 (9): 1331-4, 1995

26- WHITESIDE L.A.: Selective ligament release in total knee arthroplasty of the knee in valgus. Clin. Orthop. Relat. Res., 367: 130-40, 1999.

27- KRACKOW K.A. and MIHALKO W.M.: Flexionextension joint gap changes after lateral structure release for valgus deformity correction in total knee arthroplasty: 
A cadaveric study. J. Arthroplasty, 14 (8): 994-1004, 1999.

28- ENGH G.A.: The difficult knee: severe varus and valgus. Clin. Orthop. Relat. Res., 416: 58-63, 2003.

29- BOYER P., BOUBLIL D., MAGRINO B., MASSIN P., HUTEN D. and GUEPAR GROUP: Total knee replacement in the fixed valgus deformity using a lateral approach: Role of the automatic iliotibial band release for a successful balancing. Int. Orthop., 33 (6): 1577-83, 2009.

30- AGLIETTI P., LUP D., CUOMO P., BALDINI A. and De LUCA L.: Total knee arthroplasty using a pie-crusting technique for valgus deformity. Clin. Orthop. Relat. Res., 464: 73-7, 2007.

31- CLARKE H.D., FUCHS R., SCUDERI G.R., SCOTT W.N. and INSALL J.N.: Clinical results in valgus total knee arthroplasty with the "pie crust" technique of lateral soft tissue releases. J. Arthroplasty, 20 (8): 1010-4, 2015.

32- BRUZZONE M., RANAWAT A., CASTOLDI F., DETTONI F., ROSSI P. and ROSSI R.: The risk of direct peroneal nerve injury using the Ranawat "inside-out" lateral release technique in valgus total knee arthroplasty. J. Arthroplasty, 25 (1): 161-5, 2010.

33- BRILHAULT J., LAUTMAN S., FAVARD L. and BURDIN P.: Lateral femoral sliding osteotomy lateral release in total knee arthroplasty for a fixed valgus deformity. J. Bone Joint Surg. Br., 84 (8): 1131-7, 2002.

34- BREMER D., ORTH B.C., FITZEK J.G. and KNUTSEN A.: Briard's sagittal sliding osteotomy of the lateral condyle in total knee arthroplasty of the severe valgus knee. Oper. Orthop. Traumatol., 24 (2): 95-108, 2012.

35- MULLAJI A.B. and SHETTY G.M.: Lateral epicondylar osteotomy using computer navigation in total knee arthroplasty for rigid valgus deformities. J. Arthroplasty, 25 (1): 166-9, 2010.

36- KRACKOW K.A. and HOLTGREWE J.L.: Experience with a new technique for managing severely overcorrected valgus high tibial osteotomy at total knee arthroplasty. Clin. Orthop. Relat. Res., 258: 213-24, 2016.

37- NERCESSIAN O.A., UGWONALI O.F. AND PARK S.: Peroneal nerve palsy after total knee arthroplasty. J. Arthroplasty, 20: 1068-73, 2015.

38- SCHINSKY M.F., MACAULAY W., PARKS M.L., et al.: Nerve injury after primary total knee arthroplasty. J. Arthroplasty, 16: 1048-54, 2001.

39- ROSSI R., BRUZZONE M., BONASIA D.E., MARMOTTI A. and CASTOLDI F.: Evaluation of tibial rotational alignment in total knee arthroplasty: A cadaver study. Knee Surg. Sports Traumatol. Arthrosc., 18 (7): 889-93, 2010.

\section{الإستبدال الكامل لمفصل الركبة لحالات تقبب الركبة الوحشى}

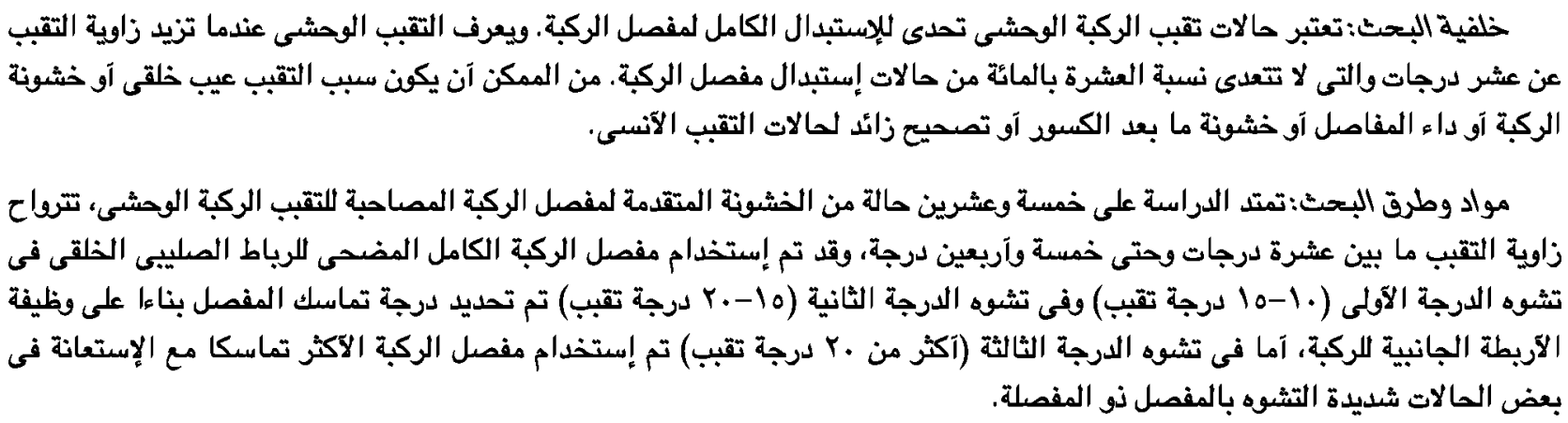

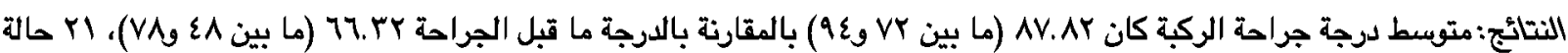

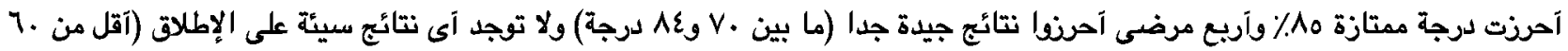

